\title{
Ultrafast Laser Machining of Micro Grooves on Stainless Steel with Spatially Optimized Intensity Distribution
}

\author{
C. Mauclair ${ }^{{ }^{1}}$, S. Landon $^{* 2}$, D. Pietroy ${ }^{* 1}$, E. Baubeau ${ }^{* 3}$, R. Stoian ${ }^{* 1}$, E. Audouard ${ }^{{ }^{* 1}}$ \\ ${ }^{*}$ Laboratoire Hubert Curien, UMR 5516 CNRS, Université de Lyon, Université Jean Monnet \\ 42000 Saint Etienne, France \\ E-mail: cyril.mauclair@univ-st-etienne.fr \\ ${ }^{*}$ Qiova, 42000 Saint Etienne, France \\ *3 Impulsion SAS, 42000 Saint Etienne, France
}

\begin{abstract}
Femtosecond laser processing enables the machining of micro-grooves by translating the focused femtosecond beam on the sample surface. Most of the time, the spatial intensity distribution on the sample is nearly Gaussian with a circular symmetry. The micro-grooves machined this way show 'classic' cross sectional profile with a ' $\mathrm{v}$ ' shape. We present here a technique to modulate the cross sectional profile of the micro-grooves in a single laser scan. The technique relies on advanced spatial beam shaping where the intensity distribution is designed to spatially modulate the pulse overlapping during the scanning process. The corresponding exposure gradient enables to control the cross sectional profile of the micro groove. We present machining results on stainless steel using ultrashort pulses $(120 \mathrm{fs}, 800 \mathrm{~nm}$ at a repetition rate of $5 \mathrm{kHz})$ where calculations based on the ablation rate clearly match the cross sectional profile of the machined micro grooves.
\end{abstract}

DOI: $10.2961 / \mathrm{jlmn} .2013 .01 .0003$

Keywords: Femtosecond machining, spatial beam shaping, wavefront modulation, laser microprocessing, ablation rate

\section{Introduction}

The specificities of femtosecond light pulses interaction with metals have been widely studied [1-3] and put forward especially in the field of micro processing. The interaction region presents a reduced thermally affected zone compared to longer irradiation sequence (nanosecond and beyond) [1]. Micro grooves with a high degree of precision can thus be realized by translating the focused femtosecond spot on the surface of the sample. The dimension of the machined structures depends on the focusing strength and the interplay of the energetic conditions and the physical properties of the irradiated material. More precisely, submicrometric dimensions beyond the optical diffraction limit are reachable by finely tuning the energetic dose around the modification threshold [4]. This unique characteristic of ultrashort lasers in micro processing of metallic surfaces has triggered research efforts to increase the efficiency of the technique to qualify it for industrial demands. For example, it has been shown that using a spatial light modulator (SLM) enables to imprint arbitrary wavefront modulation on the propagating laser wave in order to obtain userdefined intensity distributions at the focal plane of a lens [5]. In particular, demultiplication of the focal spot into several foci enables parallel processing greatly reducing the processing time and cost [6]. Efforts were also conducted on the temporal aspect of the irradiation. Recently, shaping on the picosecond scale of the light pulses has shown the possibility to increase the ablation efficiency [7]. Usually in femtosecond processing, the laser spatial intensity repartition on the sample is quasi-Gaussian having thus a circular symmetry. Consequently, the micro-grooves machined with such an energetic distribution show 'classic' cross sectional profile resembling a ' $\mathrm{v}$ '.
In this work, we present a technique allowing to design the cross sectional profile of the micro-grooves. With adequate spatial intensity distribution obtained with spatial beam shaping, the pulse overlapping during the sample translation can be modulated yielding controllable crosssectional profile of the micro-groove. Experimental results on stainless steel are presented and compared with $a$ priori topological calculations based on the accumulated energy deposition and the ablation rate.

\section{Experimental details}

A femtosecond amplified laser system (Thales Bright) operating at a repetition rate of $5 \mathrm{kHz}$ delivers $120 \mathrm{fs}$ (full width half maximum) light pulses at $800 \mathrm{~nm}$ with an average power of $2 \mathrm{~W}$ and linear polarization. As illustrated in Fig. 1, the laser beam is reflected on a SLM (Holoeye Pluto NIR) where its spatial phase undergoes a computercontrolled modulation which spatially shapes its intensity distribution in the far field (i.e here the focal plane of the focusing lens). The beam is then reduced in size by passing through a telescope $(\mathrm{T})$ which also optically conjugates the SLM plane with the entrance pupil of the focusing lens (FL) of $50 \mathrm{~mm}$-focal length. The latter focuses the ultrashort pulses on the surface of the metallic sample. In this report, common stainless steel $(316 \mathrm{~L})$ is employed. A home-made microscope sharing the focusing lens with an additional tube lens $(\mathrm{f}=250 \mathrm{~mm})$ and a two-dimensional CMOS sensor is set up for in-situ observation of the interaction zone and for monitoring the laser intensity distribution on the surface. The sample is mounted on translation stages with sub-micrometric precision. The laser energy is adjusted using half wave plate with polarizing cube and density filters (not shown). The experimental intensity dis- 


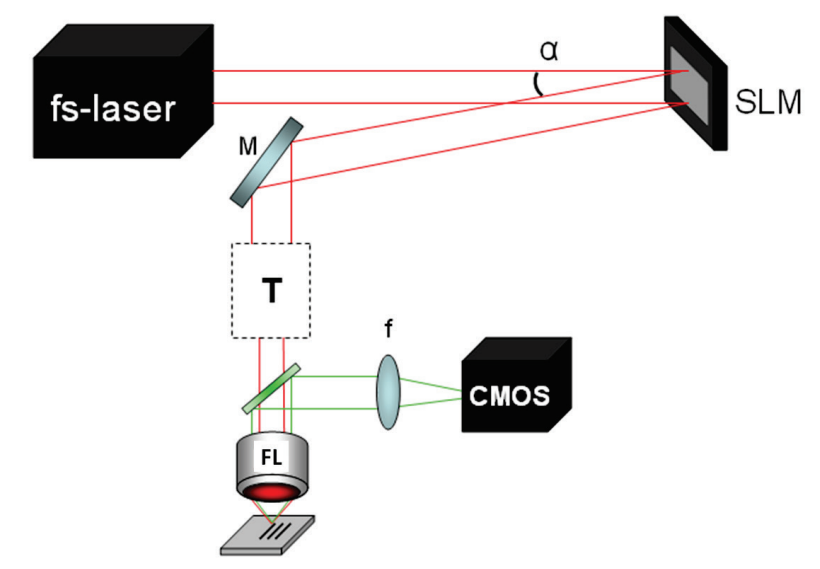

Fig. 1 Experimental set-up M: mirror, T: telescope, FL: focusing lens, $\alpha$ : reflection angle $\left(4^{\circ}\right)$, f: convergent lens of focal $f$.

tributions were measured with the two-dimensional CMOS camera. From the measured pulse energy, the fluence cartographies presented in the following were calculated and correspond to peak fluences.

Using a home-made Iterative Fourier Transform Algorithm based on the well-known Gerchberg-Saxton algorithm [5], we have calculated phase masks to generate user defined intensity profile on the sample. Two types of intensity distributions were achieved, namely discrete (multispot) and continuous profiles.

Micro grooves were machined by translating in a single scan the sample along the beam polarization during femtosecond irradiation with continuous air blowing. The lens focal plane is precisely adjusted on the surface of the sample. To evaluate the cross sectional profile of the micro grooves, the samples were immerged in an ultrasonic bath for cleaning and mechanically cut perpendicularly to the micro grooves. Then samples were immerged in a selfpolymerizing liquid and polished to reveal the micro groove cross sections which were observed under episcopic microscopy.

\section{Results and discussion}

Experimental intensity distributions are compared with theoretical scopes in Fig. 2 a) and b) showing a so-called multispot repartition were each focal spot has dimensions very close to the point spread function (PSF) of the focusing lens. The intensity distributions captured experimentally closely match the theoretical ones. From these intensity profiles, it is straightforward to estimate qualitatively the cross sectional profile of the energetic deposition on the sample surface when irradiated during translation for micro groove fabrication. For example, let us consider the intensity map in Fig. 2a) with a translation along the white arrow. If the fluence domain is situated in a linear region of the ablation rate per pulse (here we are in the gentle ablation regime i.e for fluences below $1 \mathrm{~J} / \mathrm{cm}^{2}$ on stainless steel as determined in [8]) then the depth of the micro groove is nearly proportional to the logarithm of the local fluence. Thus, projecting the intensity along the translation direction yields a profile proportional to the cross sectional profile of a micro groove machined in this direction. There, maxima of accumulated exposure correspond to maxima of local ablation, i.e the local depth of the micro groove. Fig. 2c) a)

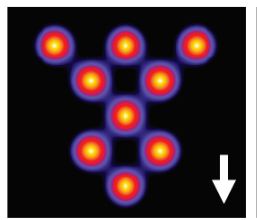

b)
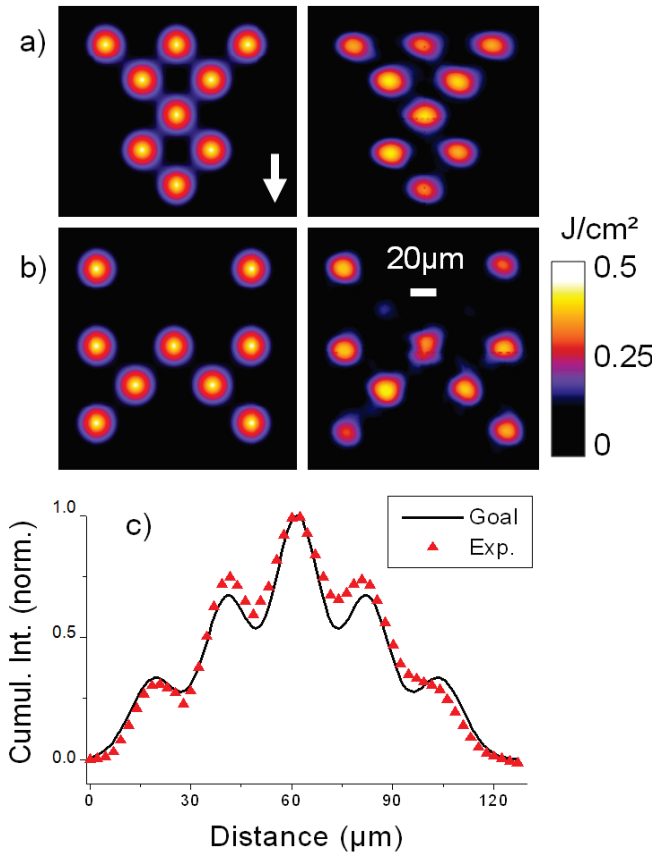

Fig. 2 a) and b) theoretical (lett) and experımental (right) intensity distributions of multispot operation in the processing focal plane obtained with phase modulation on the SLM. c) Projected theoretical (line) and experimental (triangle) intensity of a) along the white arrow which shows the sample translation axis.

contains the projection of the theoretical and experimental intensity distributions from Fig. 2a) along the white arrow, yielding a pyramidal profile where the central peak correspond to the vertical projection of the three central spots of the intensity distribution from Fig. 2a). By 'superimposing' more or less spots in the translation direction, it is possible to modulate the cross sectional profile of the micro groove.

This concept of groove machining with multispot profiles is extendable to continuous intensity distributions. Fig. 3 and 4 show examples of one spot shaping. Whereas the desired intensity distributions were uniform as well as the numerical simulations with a Fourier propagation algorithm [9], the experimental images of the energy repartition (Fig._3) show a well-known Speckle deformation [10]. To
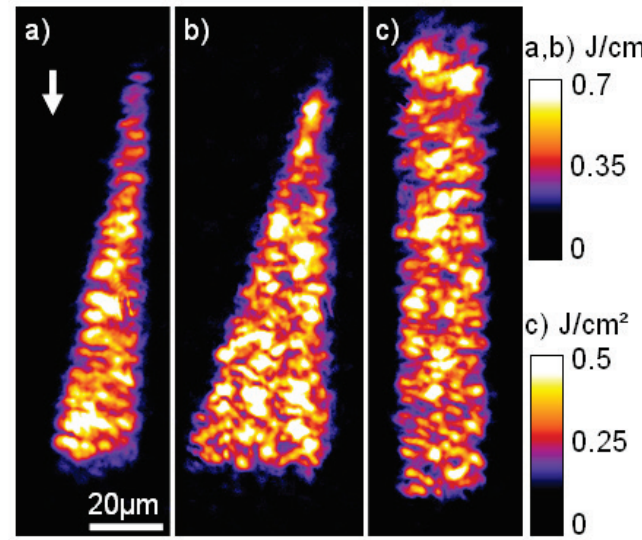

Fig. 3 Experimental intensity distributions of continuous spatial beam shaping in the processing focal plane. Examples for triangular (a and b) and rectangular (c) profiles. The white arrow shows the sample translation axis. 


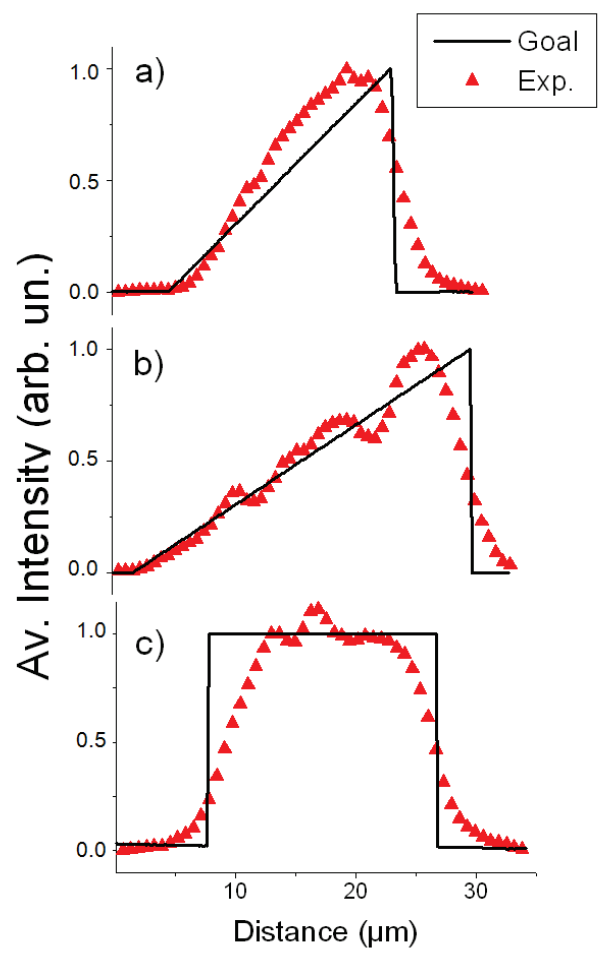

Fig. 4 Projected theoretical (line) and experimental (triangle) intensity of the distributions depicted in Fig. 3 along the white arrow. These curves a), b) and c) are proportional to the cross sectional profile of micro grooves machined with the corresponding beam profiles in Fig. 3 a) b) and c) respectively.

estimate the cross sectional profile of micro grooves machined with these profiles, we apply the concept described above. This leads to the results in Fig. 4 where the a), b) and c) curves match the Fig. 3 a), b) and c) distributions. Using this concept with uniform intensity repartition, the possibility to design the cross sectional profile of micro grooves becomes natural. In particular, Fig 4 a) and b) illustrate the possibility to generate various bending angles for the walls of the micro groove. The optical response (PSF) of the focusing lens also plays a role as it defines the maximum steepness of the intensity distribution which in turn limits the steepness of the micro groove profile. As an illustration, Fig. 4 c) shows that the expected groove profile calculated from the experimental distribution in Fig. $3 \mathrm{c}$ ) is not an ideal square with perpendicular walls (black continuous curve) but a convolution with the PSF. The depth of each groove will naturally depend on the pulse energy and overlapping during irradiation. The latter can be controlled with the sample translation speed or the number of scans (as well as the laser repetition rate) with obvious limitations due to the interaction of pulses with non-planar surfaces. Noteworthy, the method presents the advantage of averaging the Speckle deformation thanks to the overlapping during the spot translation on the sample.

It is possible to quantitatively estimate the cross sectional profile of the micro grooves with the help of experimental ablation rate curves. There, the depth removed per pulse is given as a function of the peak fluence of a Gaussian intensity distribution $[8,11]$. Whereas these curves are drawn for the peak fluence of a Gaussian distribution, it is acceptable in a first approximation to consider the relationship between the logarithm of local fluence and the ablation rate linear [12] as the fluence range covered here is in the gentle ablation region [8]. With this hypothesis, simple computer calculations enable to accurately predict the micro groove profile as illustrated by Fig. 5 following this method:

First, the two-dimensional experimental fluence cartography $F(i, j)$ has to be captured.

Second, from this array, the corresponding ablation map $A(i, j)$ is calculated for a single pulse using the linearized ablation rate curve (see Fig. 5 a)). In our case, for stainless steel $316 \mathrm{~L}$ and fluences below $1 \mathrm{~J} / \mathrm{cm}^{2}$, the ablation rate $A(\mathrm{~nm})$ is given by $A(i, j) \approx 40.9 \times \log [F(i, j)]+44$ (see [8])

Third, knowing the translation speed of the sample $v$ as well as the laser repetition rate $k$, the distance $d$ between two incoming pulses is calculated following $d=v / k$.

Last, ablation maps are added along the translation direction taking into account the displacement $d$ between each pulse. The cross sectional profile is obtained when all the ablation maps interacting on a given position have been summed (see Fig $5 \mathrm{~b}$ )). As an indication $d=0.2 \mu \mathrm{m}$ in Figs. 5 and 6, which corresponds to the overlapping of 800 pulses.

To test the robustness of the method, we realized several micro grooves on stainless steel and observed them in side view following the procedure described in the experimental section. Corresponding microscopic images are presented in Fig. 6 and compared with the calculated profile showing a very good agreement between the expected profile and the actual ones. This is true for continuous beam profiles (Fig. 6a-c) and for multispot profiles (Fig. 6d).
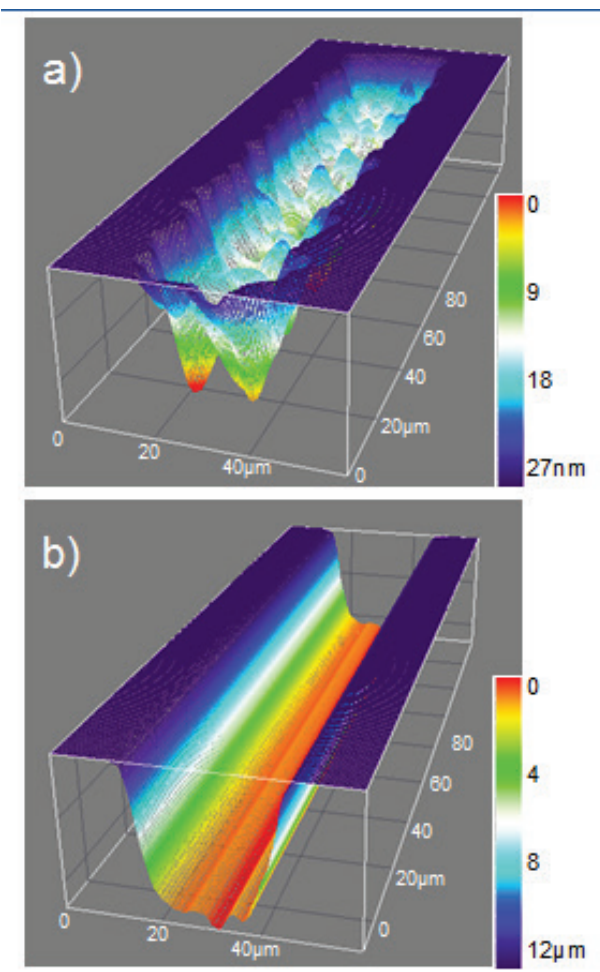

Fig. 5 a) Calculated ablation topology from a single pulse irradiation using the intensity profile from Fig. $3 \mathrm{c}$ ). b) Calculated cross sectional profile of a corresponding micro groove machined at $1 \mathrm{~mm} / \mathrm{s}$ 

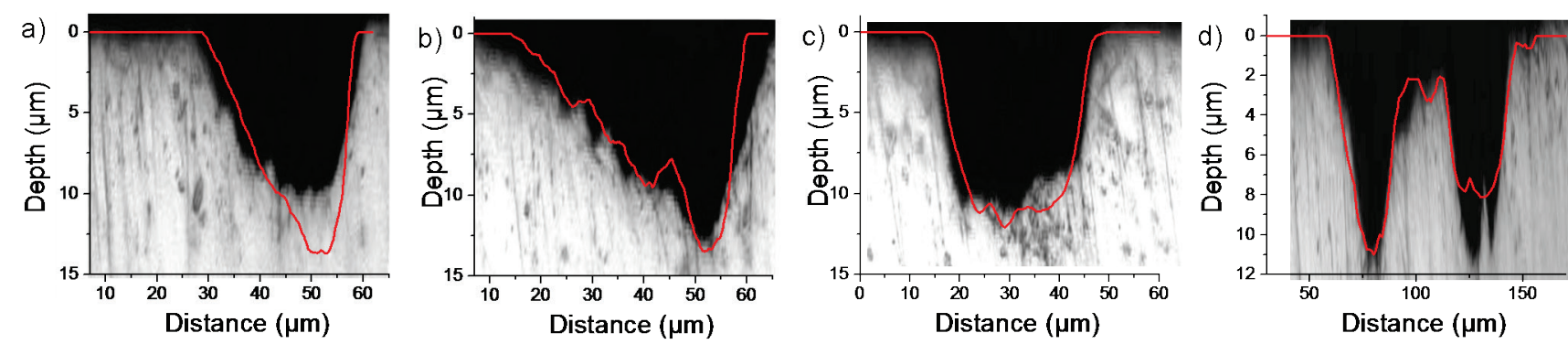

Fig. 6 Comparison of microscopic images of micro grooves on stainless steel (side view) machined at a translation speed of $1 \mathrm{~mm} / \mathrm{s}$ with a priori calculation of their cross sectional profile (red line) from the experimental fluence distribution. a), b) and c) correspond to the experimental continuous fluence distributions shown in Fig. 3 a), b) and c) respectively. d) corresponds to the multispot intensity distribution in Fig. 2 b).

Noteworthy, in Fig. 6a) and b), the number of pulses seen by a point on the sample surface varies depending on its position with respect to the triangle beam profile (Fig 3a) and b)). A maximum of overlapping is obtained for a point situated on the height of the triangle whereas no overlapping occurs for a point situated at the opposite extremity of the triangle beam profile. The cross sectional depth of the micro-groove is therefore modulated following this varying pulse overlapping. Again, the method offers reliable results as long as overlapping and energy deposition remain low enough. As shown in ref. [8], a departure from this gentle regime to higher fluences is accompanied by a deterioration of the surface roughness. This is presumably due to the formation of a liquid phase rapidly expulsed with vapor droplets followed by cooling and re-solidification. The surface topology becomes randomly modified which is outside the topic of controlled laser machining.

Current efforts are carried out to better take into account the interaction of light pulses with non-planar surfaces, for example after several photo-induced modifications. This topic is beyond the work reported here.

\section{Conclusion}

We report here on a method enabling the design of the cross sectional profile of micro-grooves machined in a single laser scan. The technique relies on spatial beam shaping where the intensity distribution is adapted to precisely control the pulse overlapping during the translation of the sample under femtosecond exposure. The corresponding exposure variations permit to modulate the cross sectional profile of the micro groove. Experimental measurements of the cross sectional profile of laser-machined micro grooves show a good agreement with the a priori calculations based on the ablation rate and pulse overlapping.

\section{Acknowledgments}

We thank S. Chapuy and T. Collange for their help on microscopy imaging. French funding OSEO ARTEMIS is acknowledged.

\section{References}

[1] B.N. Chichkov, C. Momma, S. Nolte, F. von Alvensleben and A. Tunnermann: Appl. Phys. A, 63 (1996) 109.

[2] X. Zhu, A. Naumov, D. Villeneuve and P. Corkum: Appl. Phys. A, 69 (1999) S367.

[3] J. Guedde, J. Hohlfeld, J.G. Mueller and E. Matthias: Appl. Surf. Sci., 40 (1998) 127.

[4] A. P. Joglekar, H. Liu, G. J. Spooner, E. Meyhoefer, G. Mourou and A. J. Hunt: Appl. Phys. B, 77 (2003) 25.

[5] N. Sanner, N. Huot, E. Audouard, C. Larat, J. Huignard and B. Loiseaux: Opt. Lett., 30 (2005) 1479.

[6] C. Mauclair, G. Cheng, N. Huot, E. Audouard, A. Rosenfeld, I. V. Hertel and R. Stoian: Opt. Express, 17 (2009) 3531.

[7] C. M. Liebig, P. Srisungsitthisunti, A.M. Weiner and X. Xu: Appl. Phys. A, 101 (2010) 487.

[8] P.T. Mannion, J. Magee, E. Coyne, G.M. O'Connor and T.J. Glynn: Appl. Surf. Sci., 233 (2004) 275.

[9] C. Mauclair, A. Mermillod-Blondin, S. Landon, N. Huot, A. Rosenfeld, I. V. Hertel, E. Audouard, I. Myiamoto and R. Stoian: Opt. Lett., 36 (2011) 325.

[10] W. Liu and C. Zhou: Appl. Opt., 30 (2005) 6506

[11]R. Le Harzic, D. Breitling, M. Weikert, S. Sommer, C. Foehl, S. Valette, C. Donnet, E. Audouard and F. Dausinger: Appl. Surf. Sci., 249 (2005) 321.

[12] Y. Di Maio, J. P. Colombier, P. Cazottes and E. Audouard: Optics and Lasers in Engineering, 50 (2012) 1582. 